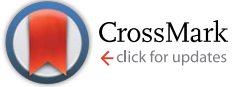

Cite this: RSC Adv., 2017, 7, 880

Received 1st November 2016 Accepted 16th November 2016

DOI: $10.1039 / c 6 r a 26133 g$

www.rsc.org/advances

\section{Oxidative decarboxylative radical trifluoromethylthiolation of alkyl carboxylic acids with silver(ı) trifluoromethanethiolate and selectfluor $\dagger$}

\author{
Bin He, Zhiwei Xiao, Hao Wu, Yong Guo, Qing-Yun Chen* and Chao Liu* \\ A straightforward silver-mediated oxidative decarboxylative radical trifluoromethylthiolation reaction of \\ aliphatic carboxylic acid is described. This reaction operates under mild conditions and allows the \\ synthesis of various valuable alkyltrifluoromethylthioethers from abundant alkyl carboxylic acids and \\ convenient nucleophilic $\mathrm{AgSCF}_{3}$ reagent. It provides a practical and efficient approach for the \\ preparation of alkyltrifluoromethylthioethers.
}

\section{Introduction}

The selective introduction of fluorine and fluorine-containing groups into organic molecules can dramatically alter their physical, chemical and biological properties. ${ }^{1}$ As a star group in organofluorine chemistry in recent years, the trifluoromethylthio group $\left(\mathrm{CF}_{3} \mathrm{~S}\right)$ has attracted much attention because of its electron-withdrawing nature, unique high lipophilicity and metabolic stability. Compounds containing $\mathrm{CF}_{3} \mathrm{~S}$ are frequently found in pharmaceuticals and agrochemicals. ${ }^{2}$ Many methods are now available to efficiently synthesize various organic substrates containing $\mathrm{CF}_{3} \mathrm{~S}$ on the basis of electrophilic, nucleophilic, radical, and oxidative direct trifluoromethylthiolation. ${ }^{3}$ Among them, more popular strategy of choice for the introduction of the $\mathrm{CF}_{3} \mathrm{~S}$ group into organic substrates is the direct formation of the $\mathrm{C}_{-} \mathrm{SCF}_{3}$ bond. Compared with numerous methods for the efficient formation of $\mathrm{C}\left(\mathrm{sp}^{2}\right)-\mathrm{SCF}_{3}$ and $\mathrm{C}(\mathrm{sp})-$ $\mathrm{SCF}_{3}$ bonds, relatively limited methods are currently available for the general and site-specific formation of various $\mathrm{C}\left(\mathrm{sp}^{3}\right)-\mathrm{SCF}_{3}$ bonds. The resulting alkyltrifluoromethylthioethers are mainly accessible by the nucleophilic, electrophilic, or radical trifluoromethylthiolation of the corresponding alkanes or olefins. For examples, a direct dehydroxytrifluoromethylthiolation of alkyl alcohols using $\mathrm{AgSCF}_{3}$ and $n-\mathrm{Bu}_{4} \mathrm{NI}$ has been reported to efficiently form $\mathrm{C}\left(\mathrm{sp}^{3}\right)-\mathrm{SCF}_{3}$ bonds. ${ }^{4}$ A direct nucleophilic trifluoromethylthiolation of various alkyl chlorides, bromides and tosylates with $\mathrm{AgSCF}_{3}$ was described in the presence of $n \mathrm{Bu}_{4} \mathrm{NI}$ or a combination of $n \mathrm{Bu}_{4} \mathrm{NI} / n \mathrm{Bu}_{4} \mathrm{NBr}^{5}$ Several electrophilic

Key Laboratory of Organofluorine Chemistry, Shanghai Institute of Organic Chemistry, Chinese Academy of Sciences, 345 Lingling Road, Shanghai 200032, China. E-mail: chaoliu@sioc.ac.cn

$\dagger$ Electronic supplementary information (ESI) available. CCDC 1484006. For ESI and crystallographic data in CIF or other electronic format see DOI: $10.1039 / \mathrm{c} 6 \mathrm{ra} 26133 \mathrm{~g}$ trifluoromethylthiolation reagents have been reported for effective trifluoromethylthiolation of organometallic nucleophilic or direct $\alpha-\mathrm{SCF}_{3}$ of carbonyl compounds. ${ }^{6}$ Recently, the direct trifluoromethylthiolation of unactivated $\mathrm{C}\left(\mathrm{sp}^{3}\right)-\mathrm{H}$ by $\mathrm{AgSCF}_{3} /$ $\mathrm{K}_{2} \mathrm{~S}_{2} \mathrm{O}_{8}$ was described via a radical pathway.

Aliphatic carboxylic acids are cheap and abundant raw materials, and have been widely used as desirable starting materials for the preparation of many important and valuable compounds with different functional groups. The fundamental transformation process involves decarboxylation with the release of traceless by-product $\mathrm{CO}_{2}$ to generate alkyl radicals, and their subsequent reactions with a variety of reagents lead to efficient formation of many functionalized compounds. This important transformation process has been applied in several elegant decarboxylative fluorinations. ${ }^{8}$ Shen and co-workers described the silver-catalyzed oxidative decarboxylative trifluoromethylthiolation of alkyl carboxylic acids with electrophilic trifluoromethylthiolating reagent $\mathbf{I}$ in an aqueous emulsion (Scheme 1a). ${ }^{8 b}$ Very recently, Glorius group reported the visiblelight-promoted decarboxylative trifluoromethylthiolation of alkyl carboxylic acids with electrophilic trifluoromethylthiolating reagent II under mild conditions (Scheme 1b). ${ }^{8 f}$ Although these methods are effective for the site-selective formation of $\mathrm{C}\left(\mathrm{sp}^{3}\right)_{-}$ $\mathrm{SCF}_{3}$, they utilize relatively expensive electrophilic trifluoromethylthiolating reagents as the $\mathrm{CF}_{3} \mathrm{~S}$ source, which are commonly prepared from readily available $\mathrm{AgSCF}_{3}$.

Recently, we developed a practical and efficient method for the direct trifluoromethylthiolation of unactivated $\mathrm{C}\left(\mathrm{sp}^{3}\right)-\mathrm{H}$ bonds by $\mathrm{AgSCF}_{3} / \mathrm{K}_{2} \mathrm{~S}_{2} \mathrm{O}_{8}$ under mild conditions. ${ }^{7 b}$ However, the regioselectivity of the reaction generally depends on the alkyl radical stability in situ formed, and several regioselective isomers are often generated in many cases. With the aim of overcoming the issue and further expanding the scope of the oxidative radical trifluoromethylthiolation methodologies, 


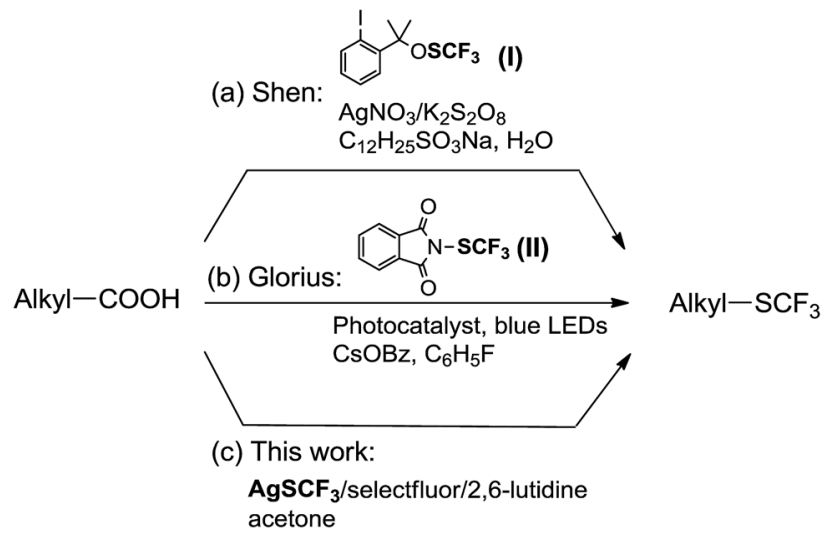

Scheme 1 Trifluoromethylthiolation of aliphatic carboxylic acids

herein we report an efficient and general site-selective preparation of alkyltrifluoromethylthioethers by means of oxidative decarboxylative radical trifluoromethylthiolation of various aliphatic carboxylic acids by $\mathrm{AgSCF}_{3} /$ selectfluor (Scheme 1c).

\section{Results and discussion}

Our study commenced by examing the oxidative decarboxylative trifluoromethylthiolation reaction of decanoic acid 1a with $\mathrm{AgSCF}_{3}$ (ref. 9) (1.0 equiv.) at $60{ }^{\circ} \mathrm{C}$ for 4 hours in the presence of different oxidants and solvents (Table 1). Unfortunately, no desired trifluoromethylthiolation product was observed with $\mathrm{K}_{2} \mathrm{~S}_{2} \mathrm{O}_{8}$ as oxidant in $\mathrm{CH}_{3} \mathrm{CN}$ (Table 1, entry 1 ). To our surprise, under similar conditions, $\mathrm{Na}_{2} \mathrm{~S}_{2} \mathrm{O}_{8}$ resulted in the formation of oxidative decarboxylative trifluoromethylthiolation products (Table 1, entry 2). However, after careful analysis and spectroscopic characterization, it was found that the products are not the desired product $\mathbf{2 a}$, but a various di-trifluoromethylthiolation isomer mixture, which is presumably due to concurrent occurrence of both direct trifluoromethylthiolation of $\mathrm{C}\left(\mathrm{sp}^{3}\right)-\mathrm{H}$ and the desired oxidative decarboxylative trifluoromethylthiolation of 1a (Scheme 2). In order to suppress the undesired direct trifluoromethylthiolation of $\mathrm{C}\left(\mathrm{sp}^{3}\right)-\mathrm{H}$, different solvents including DMSO, DMF, acetone, and $\mathrm{ClCH}_{2} \mathrm{CH}_{2} \mathrm{Cl} / \mathrm{CH}_{3} \mathrm{CN} / \mathrm{H}_{2} \mathrm{O}$ were screened. However, the reactions led to no formation of the desired products. ${ }^{10}$ When $\mathrm{AgSCF}_{3}$ was used as the limiting reagent with 5 equiv. of $\mathbf{1 a}$ in $\mathrm{CH}_{3} \mathrm{CN}$ in the presence of $\mathrm{Na}_{2} \mathrm{~S}_{2} \mathrm{O}_{8}$, a complex di-trifluoromethylthiolation isomer mixture was still obtained (Table 1 , entry 3 ).

Next, we were delighted to find that when selectfluor was used as oxidant, the desired oxidative decarboxylative monotrifluoromethylthiolation product $\mathbf{2 a}$ was obtained and no undesired di-trifluoromethylthiolation products were observed, demonstrating the successful suppression of the undesired direct trifluoromethylthiolation of $\mathrm{C}\left(\mathrm{sp}^{3}\right)-\mathrm{H}$ (Table 1 , entry 4 ). Notably, neither competitive oxidative decarboxylative fluorination reaction was observed, which was considered to presumably occur under the reaction conditions according to literature. ${ }^{8 a}$ These results demonstrate that oxidative decarboxylative trifluoromethylthiolation under the reaction
Table 1 Optimization of reaction conditions ${ }^{a}$

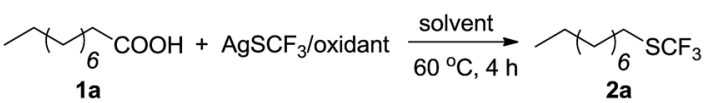

\begin{tabular}{lllll}
\hline Entry & $\mathrm{AgSCF}_{3}$ (equiv.) & Oxidant (equiv.) & Solvent & Yield $^{b}(\%)$ \\
\hline 1 & 1.0 & $\mathrm{~K}_{2} \mathrm{~S}_{2} \mathrm{O}_{8}(2.0)$ & $\mathrm{CH}_{3} \mathrm{CN}$ & 0 \\
2 & 1.0 & $\mathrm{Na}_{2} \mathrm{~S}_{2} \mathrm{O}_{8}(2.0)$ & $\mathrm{CH}_{3} \mathrm{CN}$ & $37^{c}$ \\
3 & 1.0 & $\left(\mathrm{NH}_{4}\right)_{2} \mathrm{~S}_{2} \mathrm{O}_{8}$ & $\mathrm{CH}_{3} \mathrm{CN}$ & $28^{c}$ \\
4 & 1.0 & Selectfluor $(2.0)$ & Acetone & 31 \\
5 & 1.0 & Selectfluor $(2.0)$ & $\mathrm{CH}_{3} \mathrm{CN}$ & 1 \\
6 & 1.0 & Selectfluor $(2.0)$ & DMF & 3 \\
7 & 1.0 & Selectfluor $(2.0)$ & Acetone $/ \mathrm{CH}_{3} \mathrm{CN}$ & 3 \\
8 & 1.0 & Selectfluor $(2.0)$ & Acetone/ $\mathrm{H}_{2} \mathrm{O}$ & $0^{e}$ \\
9 & 1.0 & NFSI (2.0) & Acetone & 25 \\
10 & 1.0 & FP (2.0) & Acetone & 5 \\
11 & 2.0 & Selectfluor $(2.0)$ & Acetone & 54 \\
12 & 2.0 & Selectfluor (4.0) & Acetone & 65 \\
13 & 2.0 & Selectfluor (4.0) & Acetone & $82^{f}$ \\
14 & 2.0 & Selectfluor (4.0) & Acetone & $0^{g}$
\end{tabular}

${ }^{a}$ Reaction conditions: decanoic acid ( $0.2 \mathrm{mmol}, 1.0$ equiv.), $\mathrm{AgSCF}_{3}$ (1.0-2.0 equiv.), oxidant (2.0-4.0 equiv.), and solvent $(2.0 \mathrm{~mL})$ at $60^{\circ} \mathrm{C}$ for 4 hours under Ar atmosphere. ${ }^{b}$ Yields were determined by ${ }^{19} \mathrm{~F}$ NMR spectroscopy with benzotrifluoride as the internal standard. ${ }^{c} \mathrm{~A}$ di-trifluoromethylthiolation isomer mixture was obtained. ${ }^{d}$ Acetone/ $\mathrm{CH}_{3} \mathrm{CN}(1: 1 \mathrm{v} / \mathrm{v})$ was used. ${ }^{e}$ Acetone $/ \mathrm{H}_{2} \mathrm{O}(4: 1 \mathrm{v} / \mathrm{v})$ was used. ${ }^{f} 2,6-$ Lutidine (2.0 equiv.) was used as ligand. ${ }^{g} \mathrm{CuSCF}_{3}$ was used instead of $\mathrm{AgSCF}_{3}$.

conditions is the predominant process presumably because selectfluor would not activate the $\mathrm{C}\left(\mathrm{sp}^{3}\right)-\mathrm{H}$ to give the corresponding key $\mathrm{C}\left(\mathrm{sp}^{3}\right)$-centered radical intermediate. Among several common reaction solvents we screened, acetone was the best one and provided 31\% yield of the desired product 2 a (Table 1, entries 4-7). It is worth noting that water was harmful for the reaction and resulted in no formation of the desired product (Table 1, entry 8). Then various oxidants such as $N$ fluorobenzenesulfonimide (NFSI), and 1-fluoropyridium tetrafluoroborate $(\mathrm{PF})$ were further tested, it turned out that worse results were observed (Table 1, entries 9 and 10). By changing the amount of $\mathrm{AgSCF}_{3} /$ selectfluor, the yield of the desired product 2 a was further improved to $65 \%$ (Table 1, entries 11 and 12). The influence of several ligands were also estimated, 2,6lutidine gave the best yield of $82 \%$ (Table 1, entry 13), which might coordinate with active $\mathrm{Ag}$ species to enhance their stability during the reaction. ${ }^{10}$ Furthermore, the replacement of $\mathrm{AgSCF}_{3}$ with $\mathrm{CuSCF}_{3}$ led to no formation of the desired product, demonstrating the key role of the free silver cation for the reaction (Table 1, entry 14). All these experimental results indicate that a combination of $\mathrm{AgSCF}_{3}$ (2.0 equiv.)/selectfluor (4.0 equiv.)/2,6-lutidine (2.0 equiv.) in acetone at $60{ }^{\circ} \mathrm{C}$ for 4

$$
\sim_{6} \mathrm{COOH} \frac{\mathrm{AgSCF}_{3} / \mathrm{Na}_{2} \mathrm{~S}_{2} \mathrm{O}_{8}}{\mathrm{CH}_{3} \mathrm{CN}, 60^{\circ} \mathrm{C}, 4 \mathrm{~h}} \underset{\mathrm{F} 3 \mathrm{CS}}{\longrightarrow} \underset{6}{\mathrm{SCF}_{3}}
$$

Scheme 2 Competitive oxidative trifluoromethylthiolation reactions of 1 . 
hours is established as the optimal conditions for oxidative decarboxylative trifluoromethylthiolation reactions of various aliphatic carboxylic acids.

With the optimal conditions in hand, we then explored the generality of the oxidative decarboxylative trifluoromethyl thiolation reactions with a variety of aliphatic carboxylic acids (Scheme 3). It was found that various aliphatic carboxylic acids including primary, secondary and tertiary carboxylic acids are all suitable substrates for the oxidative decarboxylative trifluoromethylthiolation reactions. For example, a number of primary alkyl carboxylic acids underwent smooth oxidative decarboxylative trifluoromethylthiolation to give the desired alkylthifluormethylthioethers in isolated yields of $48-84 \%$ (2a-k). Secondary aliphatic carboxylic acids could also be subjected to the reactions to provide the corresponding products in modest to good yields (2l-q). When tertiary alkyl carboxylic acids were employed as the substrates, the desired oxidative decarboxylative trifluoromethylthiolation products were generated in acceptable yields $(2 \mathbf{r}, \mathbf{s})$. It should be mentioned that reduced reaction time should be applied to the aliphatic carboxylic acids $\mathbf{1} \mathbf{j}$ and $\mathbf{1 l}$ since significant decrease in the yields of the corresponding desired trifluoromethylthiolation products was observed under the optimal reaction conditions, ${ }^{10}$ which might be due to the instability of the corresponding products $2 \mathbf{j}$ and $\mathbf{2 l}$ under the reaction

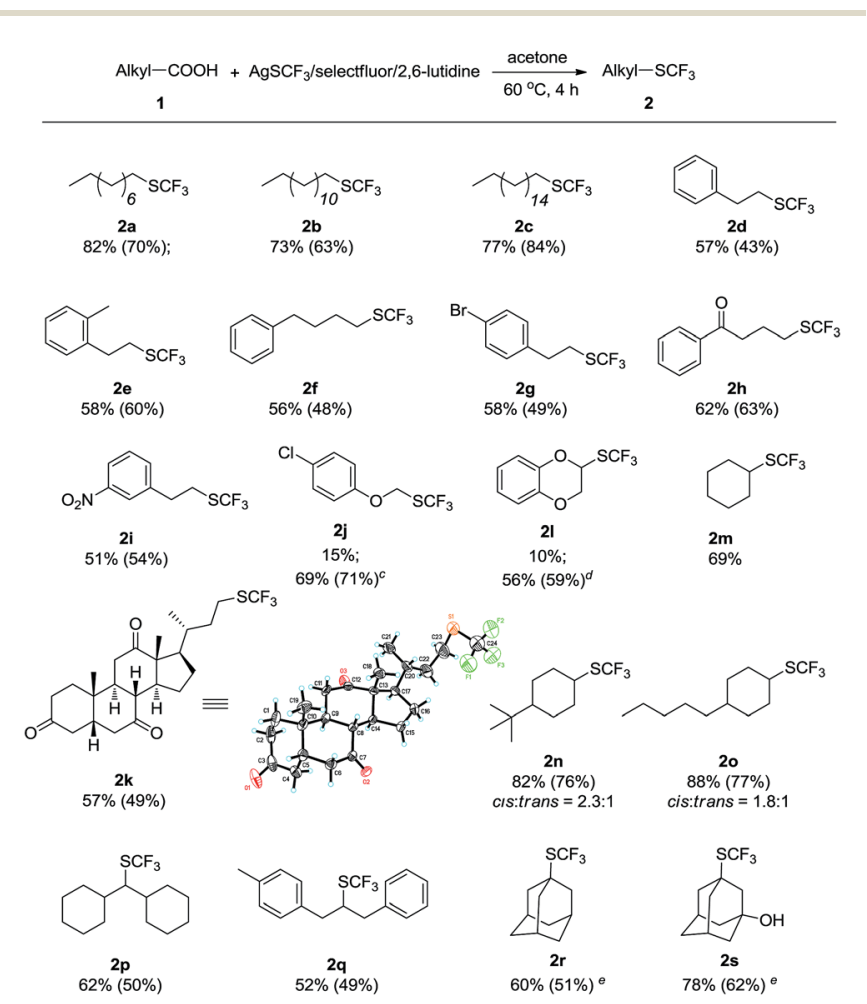

Scheme 3 Scope of oxidative decarboxylative trifluoromethylthiolation reactions of various aliphatic carboxylic acid$\mathrm{s}^{a, b}$ a Reaction conditions: $1(0.5 \mathrm{mmol}), \mathrm{AgSCF}_{3}(1.0 \mathrm{mmol})$, selectfluor $(2.0 \mathrm{mmol})$, and 2,6-lutidine $(1.0 \mathrm{mmol})$ in acetone $(1 \mathrm{~mL})$ at $60{ }^{\circ} \mathrm{C}$ for $4 \mathrm{~h}$ under Ar atmosphere. ${ }^{b}$ Yields were determined by ${ }^{19} \mathrm{~F}$ NMR spectroscopy with trifluorotoluene as internal standard. Yields of isolated products are given in parentheses. ${ }^{c}$ Reaction time: $2 \mathrm{~h}$. ${ }^{d}$ Reaction time: 15 min. ${ }^{e} 1$ (1.5 mmol), $\mathrm{AgSCF}_{3}(0.5 \mathrm{mmol})$, selectfluor $(1.0 \mathrm{mmol})$, and 2,6 -lutidine $(0.5 \mathrm{mmol})$ were used.

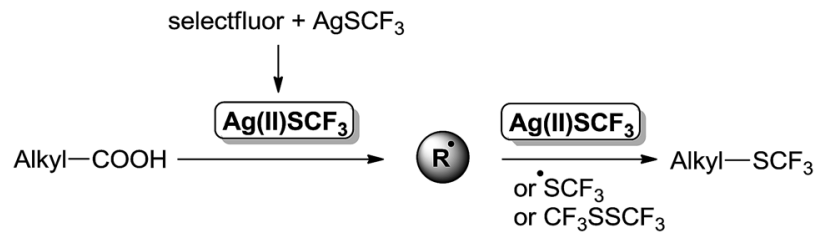

Scheme 4 Proposed mechanism.

conditions. With regard to the potential of the reaction in latestage functionalization of valuable synthetic intermediates, a structurally complicated biologically active steroid derivative was applied to the oxidative decarboxylative trifluoromethylthiolation reaction and the desired product $\mathbf{2 k}$ was produced smoothly in good isolated yield. Its structure was unambiguously assigned by various spectral analysis, including X-ray single-crystal analysis.

To gain some understanding on the reaction pathway, a radical inhibitor (hydroquinone) or a radical scavenger (2,2,6,6-tetramethyl-1-piperidinyloxy, TEMPO) were added to the reaction of 1a, and a sharp decrease in the yield was observed for both cases. ${ }^{10}$ These preliminary results combined with previous reports on silver-mediated oxidative decarboxylative functionalization ${ }^{7,8 a, b}$ indicate that the reaction proceeds through a radical pathway. ${ }^{10}$ First, $\mathrm{AgSCF}_{3}$ is oxidized by selectfluor to generated $\mathrm{Ag}(\mathrm{II}) \mathrm{SCF}_{3}$ intermediate, $\mathrm{CF}_{3} \mathrm{~S}$ radical or $\mathrm{CF}_{3} \mathrm{SSCF}_{3}$. Ag(II) combines with alkyl carboxylic acids to generate the corresponding alkyl radical via decarboxylation pathway. Then, the produced alkyl radical attacks a $\mathrm{CF}_{3} \mathrm{~S}$ radical, $\mathrm{CF}_{3} \mathrm{SSCF}_{3}$, or abstracts the $\mathrm{CF}_{3} \mathrm{~S}$ ligand of $\mathrm{Ag}(\mathrm{II}) \mathrm{SCF}_{3}$ intermediate to provide the desired oxidative decarboxylative trifluoromethylthiolation product (Scheme 4).

\section{Conclusions}

In conclusion, we developed a practical and effective silvermeditated oxidative decarboxylative trifluoromethylthiolation reaction of aliphatic carboxylic acids using nucleophilic $\mathrm{AgSCF}_{3}$ as $\mathrm{CF}_{3} \mathrm{~S}$ source and selectfluor as the oxidant. The reaction can be applied to primary, secondary and tertiary alkyl carboxylic acids under mild conditions and is tolerant of many functional groups. It represents an alternative approach for site-selective construction of $\mathrm{C}\left(\mathrm{sp}^{3}\right)-\mathrm{SCF}_{3}$ bonds.

\section{Acknowledgements}

The authors gratefully acknowledge the financial support from the National Basic Research Program of China (2012CB821600), the National Natural Science Foundation of China (21032006, 21302207, 21421002).

\section{Notes and references}

1 (a) J.-P. Bégué and D. Bonnet-Delpon, Bioorganic and Medicinal Chemistry of Fluorine, John Wiley \& Sons, New Jersey, 2008; (b) I. Ojima, Fluorine in Medicinal Chemistry and Chemical Biology, Wiley-Blackwell, Hong Kong, 2009; (c) W. K. Hagmann, J. Med. Chem., 2008, 51, 4359; (d) 
S. Purser, P. R. Moore, S. Swallow and V. Gouverneur, Chem. Soc. Rev., 2008, 37, 320; (e) K. Müller, F. Faeh and F. Diederich, Science, 2007, 317, 1881.

2 J. Wang, M. Sánchez-Roselló, J. L. Aceña, C. D. Pozo, A. E. Sorochinsky, S. Fustero, V. A. Soloshonok and H. Liu, Chem. Rev., 2014, 114, 2432.

3 For selected reviews, see: $(a)$ X.-H. Xu, K. Matsuzaki and N. Shibata, Chem. Rev., 2015, 115, 731; (b) F. Toulgoat, S. Alazet and T. Billard, Eur. J. Org. Chem., 2014, 2415; (c) X. Shao, C. Xu, L. Lu and Q. Shen, Acc. Chem. Res., 2015, 48, 1227; (d) L. Chu and F.-L. Qing, Acc. Chem. Res., 2014, 47, 1513; (e) G. Landelle, A. Panossian, S. Pazenok, J.-P. Vors and F. Leroux, Beilstein J. Org. Chem., 2013, 9, 2476; (f) T. Liang, C. Neumann and T. Ritter, Angew. Chem., Int. Ed., 2013, 52, 8214; (g) V. Boiko, Beilstein J. Org. Chem., 2010, 6, 880; (h) K. Zhang, X. Xu and F. Qing, Chin. J. Org. Chem., 2015, 35, 556; (i) H. Zheng, Y. Huang and Z. Weng, Tetrahedron Lett., 2016, 57, 1397; (j) H. Chachignon and D. Cahard, Chin. J. Chem., 2016, 34, 445.

4 J.-B. Liu, X.-H. Xu, Z.-H. Chen and F.-L. Qing, Angew. Chem., Int. Ed., 2015, 54, 897.

5 C. Xu, Q. Chen and Q. Shen, Chin. J. Chem., 2016, 34, 495.

6 For representative examples, see: (a) X. Shao, X. Wang, T. Yang, L. Lu and Q. Shen, Angew. Chem., Int. Ed., 2013, 52, 3457; (b) E. V. Vinogradova, P. Müller and S. L. Buchwald, Angew. Chem., Int. Ed., 2014, 53, 3125; (c) X. Wang, T. Tang, X. Cheng and Q. Shen, Angew. Chem., Int. Ed., 2013, 52, 12860; (d) C. Xu, B. Ma and Q. Shen, Angew. Chem., Int. Ed., 2014, 53, 9316; (e) X. Shao, T. Liu, L. Lu and Q. Shen, Org. Lett., 2014, 16, 4738; $(f)$ F. Baert,
J. Colomb and T. Billard, Angew. Chem., Int. Ed., 2012, 51, 10382; $(g)$ T. Bootwicha, X. Liu, R. Pluta, I. Atodiresei and M. Rueping, Angew. Chem., Int. Ed., 2013, 52, 12856; (h) Y. D. Yang, A. Azuma, E. Tokunaga, M. Y. Amasaki, M. Shiro and N. Shibata, J. Am. Chem. Soc., 2013, 135, 8782; (i) X. L. Zhu, J. H. Xu, D. J. Cheng, L. J. Zhao, X. Y. Liu and B. Tan, Org. Lett., 2014, 16, 2192; (j) S. Alazet, L. Zimmer and T. Billard, J. Fluorine Chem., 2015, 171, 78.

7 (a) S. Guo, X. Zhang and P. Tang, Angew. Chem., Int. Ed., 2015, 54, 4065; (b) H. Wu, Z. Xiao, J. Wu, Y. Guo, J.-C. Xiao, C. Liu and Q.-Y. Chen, Angew. Chem., Int. Ed., 2015, 54, 4070.

8 For recent reports, see: (a) F. Yin, Z. Wang, Z. Li and C. Li, J. Am. Chem. Soc., 2012, 134, 10401; (b) F. Hu, X. Shao, D. Zhu, L. Lu and Q. Shen, Angew. Chem., Int. Ed., 2014, 53, 6105; (c) M. Rueda-Becerril, O. Mahe, M. Drouin, M. B. Majewski, J. G. West, M. O. Wolf, G. M. Sammis and J. F. Paquin, J. Am. Chem. Soc., 2014, 136, 2637; (d) S. Ventre, F. R. Petronijevic and D. W. C. MacMillan, J. Am. Chem. Soc., 2015, 137, 5654; (e) H. Huang, K. Jia and Y. Chen, Angew. Chem., Int. Ed., 2015, 54, 1881; (f) L. Candish, L. Pitzer, A. Gómez-Suárez and F. Glorius, Chem.-Eur. J., 2016, 22, 4753.

9 The exact molecular formula of silver(I) trifluoromethanethiolate prepared according to the literature (G. Teverovskiy, D. S. Surry and S. L. Buchwald, Angew. Chem., Int. Ed., 2011, 50, 7312) is $3 \mathrm{AgSCF}{ }_{3} \cdot \mathrm{CH}_{3} \mathrm{CN}^{7 b}$ For simplicity, we used $\mathrm{AgSCF}_{3}$ instead of $3 \mathrm{AgSCF}_{3} \cdot \mathrm{CH}_{3} \mathrm{CN}$ in the text.

10 See the ESI for details. $\dagger$ 